\title{
Clinical associates in South Africa
}

In 2008, the National Department of Health (NDoH) in South Africa (SA) introduced a new health profession of clinical associates (ClinAs). This was a response to a chronic shortage of healthcare workers, especially doctors. ClinAs are professional members of the healthcare system with the necessary skills and knowledge to function effectively mainly in primary healthcare settings such as clinics, community health centres (CHCs) and district hospitals. ClinAs are registered under the Health Professions Council of South Africa, with their scope of practice allowing them to do the following (inter alia): ${ }^{[1]}$

- conduct consultations (history taking and physical examination)

- order and interpret investigations (e.g. electrocardiograms, laboratory tests, X-rays)

- diagnose and treat common conditions

- perform procedures (e.g. lumbar puncture, intercostal drain)

- assist in surgery (e.g. caesarean section)

- provide patient education and counselling

- make appropriate admissions, discharges and referrals

- prescribe medicines for common and important conditions

- issue sick certificates.

ClinAs are currently trained for 3 years (Bachelor of Clinical Medical Practice) at the University of Pretoria, the University of the Witwatersrand and Walter Sisulu University. These three universities produced 1070 qualified ClinAs by the end of 2018, the majority of whom work in the public sector.

ClinAs could be a solution in addressing the chronic shortage of doctors in SA and dealing with this country's quadruple burden of disease by advancing promotive, preventive and curative health services at all levels of district and primary healthcare. The findings of Moodley et al. ${ }^{[2]}$ showed that $57 \%$ of ClinA students intended to work in rural areas, in contrast to the findings of Burch et al. ${ }^{[3]}$ that $4.8 \%$ of medical students were interested in practising in rural settings beyond their mandatory training and bursary obligations. While the shortage of doctors particularly affects the rural areas, there is also a shortage in the public sector in general, and according to a 2016 survey, $80 \%$ of ClinAs are already serving district health services at clinic, $\mathrm{CHC}$ and hospital level.

A family medicine registrar in Mpumalanga working with ClinAs described them as follows (unpublished communication, October 2017): 'Academic wise they have more practical ways of approaching a case, they stimulate and challenge me academically as they have a lot of information, and their level of practice is very close to that of a general practitioner. They are skilled in making a diagnosis and managing patients.' This is a general feeling among many doctors, nurses and other health professionals who have worked with ClinAs.

SA is in need of both short- and long-term solutions to its current health crisis. Training of ClinAs only takes 3 years, and their impact is felt by the system as early as their first year of training. When doing practical studies in health facilities, medical students not only learn, they also contribute to delivery of health services. ${ }^{[4]}$ Training of ClinAs is more practically based, e.g. a 3rd-year ClinA is expected to work 972 normal hours and 272 after-hours a year. This undoubtedly adds value in the health workforce.

Most ClinAs are recruited from socially disadvantaged areas, so the profession provides educational and professional opportunities for young South Africans, thus addressing dire health workforce shortages in particular and youth unemployment in general.

The World Health Organization's Global Strategy on Human Resources for Health: Workforce 2030 acknowledges mid-level health workers as having a critical role to play across all service delivery priorities. In the USA, a high-income country, there are 238 physician assistant (PA) programmes. ${ }^{[5]}$ The number of PAs (known as ClinAs in SA) at primary healthcare level has been growing in the USA, with more than 100000 PAs currently practising there. The number of universities training PAs in the UK went up from less than five in 2012 to 35 in 2018. Many authors have described clinical officers as being part of the backbone of the health system in countries such as Malawi, Kenya and Zambia.

From this information, it is clear that low-, middle- and high-income countries are all in need of ClinAs. SA is no exception, and looking at the high burden of disease in this country, there is undoubtedly a need for a sustainable, affordable and scalable approach to addressing the Human Resources for Health shortage.

Ngcobo et al.${ }^{[6]}$ concluded that ClinAs perform circumcisions at a clinical standard that is comparable to circumcisions performed by doctors. They reported that ClinAs performed $88.66 \%$ of circumcisions in Tshwane. ${ }^{[6]}$ This procedure was used as an example to demonstrate that the quality of tasks shared with ClinAs is not compromised.

For the past 11 years, the NDoH has not shown significant support of the profession of ClinAs. When two national task teams were tasked to look into the challenges, one did not do the work, and the other made a report with recommendations on addressing challenges in 2017 - 2 years down the line, it still has not been adopted by the NDoH. When the profession of ClinAs was introduced, there was no scope of practice or job description, two things that are essential in determining an appropriate salary level for all professions. This lack resulted in ClinAs being underpaid and overworked. There is currently no well-defined career path for them, which is not good for a profession dominated by the youth. ${ }^{[2]}$ There is a risk that ClinAs will leave their profession because of these challenges. Only three of the eight medical schools in SA are training ClinAs, and there is no obvious reason for what is holding other universities back.

It is clear that the profession of ClinAs has the potential to change the face of the SA health system, but there is currently no political will to advance it. More work needs to be done in addressing challenges, advertising and advocating this profession.

\section{Sanele Ngcobo}

Department of Family Medicine, School of Medicine, Faculty of Health Sciences, University of Pretoria, South Africa sanele.ngcobo@up.ac.za

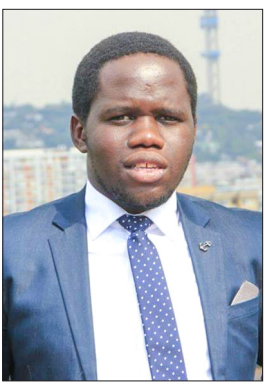

1. South Africa. Health Professions Act, 1974 (Act No. 56 of 1974). Regulations defining the scope of practice of clinical associates. Government Gazette No. 40414, 2016. https://www.hpcsa.co.za/Uploads/edito UserFiles/downloads/medical_dental/regulations/gg40414_nn1390.pdf (accessed 2 September 2019). 2. Moodley SV, Wolvaardt L, Louw M, Hugo J. Practice intentions of clinical associate students at the University of Pretoria, South Africa. Rural Remote Health 2014;14:2381.

3. Burch V, McKinley D, van Wyk J, et al. Career intentions of medical students trained in six sub-Saharan African countries. Educ Health (Abingdon) 2011;24(3):614.

Atuyambe LM, Baingana RK, Kibira SP, et al. Undergraduate students' contributions to health service delivery through community-based education: A qualitative study by the Mesau Consortium in Uganda. BMC Med Educ 2016;16(1):123.

5. ARC-PA (Accreditation Review Commission on Education for the Physician Assistant, Inc.) Program accreditation status. 2019. http://www.arc-pa.org/accreditation/accredited-programs/ (accessed 2 September 2019).

6. Ngcobo S, Wolvaardt JE, Bac M, Webb E. The quality of voluntary medical male circumcision done by midlevel workers in Tshwane District, South Africa: A retrospective analysis. PLoS One 2018;13(1):e0190795. https://doi.org/10.1371/journal.pone.0190795 